\title{
ARQUIVOS DE UEURO-PSIQUIATRIA
}

\section{LESOOES DA PAREDE DA ARTERIA CAROTIDA DA COBAIA APOS CLAMPAGEM TEMPORARIA COM DESTAQUE PARA A REDE DE VASA VASORUM}

\author{
BENEDICTO OSCAR COLLI* \\ SYLVIO DE VERGUEIRO FIORJAZ ** \\ AFFONSO LUIZ F'ERREIRA ***
}

O desenvolvimento progressivo de microclampes para a oclusão temporária de vasos ou para a oclusão definitiva de aneurismas desempenhou importante papel no recente avanço da microcirurgia vascular cerebral. Apesar da evolução tecnológica na confecção dos microclampes a oclusão temporária de vasos não é um procedimento completamente inócuo.

Estudos recentes realizados em pequenas artérias de animais, ocluidas temporariamente com vários tipos de microclampes e observadas ao microscópio eletrônico, mostraram alterações importantes da parede vascular no local da clampagem 12,15,22,30,32,37,41. Estas alterações foram lesões no endotélio vascular, que variaram desde achatamento e edema até a sua ruptura e lesões da lâmina elástica interna e da camada média, que ocasionam a formação de dilatação local. Estas lesões dependem do tipo de microclampe, do tempo de duração da oclusão e da distância entre o vaso ocluido e o fulcro do microclampe 12,32 e podem desempenhar um papel importante na gênese da trombose e oclusão destas pequenas artérias.

Em resumo, podemos dizer que as alterações observadas nas paredes das artérias ocluidas temporariamente dependem de dois fatores. Um deles é intrínseco ao microclampe e é representado pela pressão que ele exerce sobre o vaso, a qual resulta das suas características físicas 10 e o outro é o tempo de oclusão a que é submetido o vaso. Em virtude destas observações nos indagamos o que deveria acontecer à nutrição destes vasos ocluidos temporariamente, uma vez que seus vasa vasorum são ocluidos concomitantemente. A literatura consultada revelou-se escassa.

Trabalho do Departamento de Cirurgia, Ortopedia e Traumatologia e do Departamento de Morfologia da Faculdade de Medicina de Ribeirão Prêto, Universidade de São Paulo: * Médico Assistente de Neurocirurgia do Hospital das Clínicas da Faculdade de Medicina de Ribeirão Prêto; ** Professor Titular de Neurocirurgia; *** Professor Titular de Morfologia. Trabalho apresentado no XIX Congresso Latino-americano de Neurocirurgia realizado em Guayaquil, Equador, 1981. 
A primeira descrição dos vasa vasorum data, segundo Marzoli e col. 26, de 1935 mas, somente após o uso de métodos especiais de injeção, diafanização, histoquímica e raio-x $4,6,7,8,11,28,29,33,34,35,36,38,42,44$, é que foi evidenciada a rede de vasa vasorum adventicial muitọ desenvolvida.

A irrigação das paredes arteriais é feita através da difusão do sangue circulante na luz das artérias e por vasos originados em uma rede externa proveniente de ramos colaterais da própria artéria e de ramos colaterais de artérias limitrofes, concentrando-se esta rede de vasa vasorum na adventícia e no terço externo da camada média 9,16,18,26,29. Em artérias com paredes finas a irrigação interna (difusão do sangue a partir da luz), é muito importante enquanto que a rede de vasa vasorum adventicial passa a assumir maior importância conforme a espessura da parede seja maior 18,26. Linsbach (1962, citado por Gozzetti \& Vio 16) sugere que a nutrição da parede vascular seria o problema fundamental da sua patologia e daí a importância do conhecimento dos vasa vasorum para o embasamento teórico da cirurgia vascular.

Vários aspectos funcionais e patológicos dos vasa vasorum de grandes vasos foram abordados em trabalhos experimentais por diferentes autores. A adventiciectomia realizada por vários métodos ocasiona lesões na túnica média dos vasos segundo alguns autores 14,21,33. Dahl e col. 9 não observaram estas lesões a não ser que a adventiciectomia fosse executada até o ponto de ruptura iminente do vaso. Gozzetti e col. 18 realizaram adventiciectomia parcial da aorta de cães e observaram que em seguida ocorre uma reação característica dos vasa vasorum no segmento desprovido de adventícia e no segmento circundante, representada por ectasia e neoformação vascular intensa, para compensar a nutrição da parede desprovida de adventícia. Vio e col. 43 observaram que a destruição dos vasa vasorum por lesão da adventícia é um dos fatores determinantes de complicações nas suturas arteriais, pois a isquemia verificada na área prejudica a troca hemato-tissular, que é indispensável para dominar a eventual reação inflamatória local. Vários autores $5,27,28,40,44$ estudaram a evolução da vascularização de enxertos autógenos venosos e arteriais e observaram que a circulação no vaso transplantado restabelece-se precocemente. Segundo alguns autores $17,19,24,25,28$, os vasa vasorum desempenham um papel fundamental no início e na evolução da organização de próteses interpostas entre segmentos arteriais e venosos.

Em virtude da importância dos vasa vasorum nas cirurgias vasculares, elaboramos este trabalho com a finalidade de realizar um estudo morfológico comparativo dos vasa vasorum de artérias de pequeno calibre (com diâmetros de 1 a $2 \mathrm{~mm}$ ), em condições normais, após dissecção e isolamento cirúrgico, após oclusão temporária do vaso com microclampes utilizados em microneurocirurgia e eventuais alterações da parede vascular no local da clampagem.

\section{MATERIAL E METODOS}

Foram utilizadas 60 cobaias (Cavia porcellus) adultas, de ambos os sexos, com peso variando de 500 a 1.000 gramas, cujas artérias carótidas comuns apresentaram diâmetros 
externos de 1 a $2 \mathrm{~mm}$. A escolha da cobaia para a realização deste estudo foi devida à observação experimental que após a dissecção das suas artérias carótidas, prévia à oclusão temporária, não ocorre desorganização dos seus vasa vasorum. Esta desorganização fora observada em outros animais e prejudicava muito a visualização dos vasa vasorum.

Foram utilizados microclampes empregados rotineiramente para a oclusão temporária de pequenos vasos (Biemer - FD 561*) e para a oclusão definitiva de aneurismas intracranianos (Yasargil - FD $593 *$ e Medizin **). O microclampe de Biemer exerce uma pressão de aproximadamente $31 \mathrm{~g} / \mathrm{mm} 2$ quando aplicado em vasos de $1 \mathrm{~mm}$ de diâmetro e o microclampe de Yasargil exerce uma pressão de aproximadamente 71 a $92 \mathrm{~g} / \mathrm{mm} 2$ nas mesmas condições (especificação do fabricante); para o microclampe Medizin não obtivemos especificação da pressão exercida.

Para podermos estabelecer uma comparação entre os microclampes realizamos a medida das suas forças de abertura, que indiretamente correspondem às pressões exercidas pelas suas lâminas empregando o método descrito por Sugita e col. 39, modificado por Richling e col. 32. O peso suficiente para separar as lâminas do microclampe a uma distância de $1 \mathrm{~mm}$ no local correspondente à área de contato das lâminas com o vaso a ser clampado foi definido como a forca de abertura do microclampe. Este local foi estabelecido a 6,5 mm do fulcro para os microclampes de Biemer e Medizin e a $7 \mathrm{~mm}$ para o microclampe de Yasargil. As forças de abertura obtidas representam a média das três medições consecutivas e foram respectivamente 51, 148 e 254 gramas para os microclampes de Biemer, Yasargil e Medizin.

Os 60 animais foram divididos $\epsilon \mathrm{m} 10$ grupos experimentais e nestes grupos a oclusão dos vasos foi efetuada variando-se o tipo de microclampe, o tempo de duração da clampagem e o tempo decorrido entre a clampagem e a contrastação dos vasa vasomum. No grupo 1 (controle), 6 animais foram submetidos à dissecção da artéria carótida comum direita e em seguida o sistema vascular foi injetado conforme o método descrito ulteriormente. Nos outros 9 grupos os animais foram submetidos à dissecção e clampagem temporária da artéria carótida comum direita da maneira que segue: Grupos 2 (5 animais), 4 (8 animais) e 6 (5 animais) - artérias clampadas por 10 minutos, respectivamente com microclampes de Biemer, Yasargil e Medizin em cada grupo; Grupos 3 (5 animais), 5 (8 animais) e 7 (5 animais) - artérias clampadas por 30 minutos, respectivamente com microclampes de Biemer, Yasargil e Medizin em cada grupo.

Os animais dos grupos 2 a 7 foram injetados 10 minutos após o restabelecimento do fluxo na artéria (grupos com sacrifício precoce).

Grupos 8 (5 animais), 9 (7 animais) e 10 (5 animais) - artérias clampadas por 30 minutos, respectivamente com microclampes de Biemer, Yasargil e Medizin em cada grupo. Os animais destes grupos foram injetados 30 dias após a clampagem (grupos com sacrificio tardio).

Todos os animais foram operados com auxílio do microscópio cirúrgico e com técnica microcirúrgica. Os animais foram anestesiados com injeção subcutânea de etil

* Aesculap-Werke AG. Tuttlingen. Federal Republic of Germany.

** Medizin Aparelhos Cirúrgicos Ltda., Belo Horizonte, Brasil. 
(1 metil-butil) barbiturato sódico (Nembutal*), na dose de $15 \mathrm{mg}$ por quilo de peso corporal e as regiões das incisões cirúrgicas foram infiltradas com cloridrato de lidocaina ** a $1 \%$.

Mediante incisão cervical mediana um segmento de $1 \mathrm{~cm}$ da artéria carótida comum direita foi exposto, com cuidado para preservação da adventícia. Em seguida a artéria foi clampada na altura do cruzamento dos músculos esternocleidomastóideo e esternohióideo conforme referido nos grupos experimentais. Nos animais que tiverem sacrifício tardio a incisão cervical foi suturada em plano único, após a clampagem. Durante as disseç̧ões os vasos foram umedecidos com solução de cloridrato de lidocaina a $1 \%$ para evitar vasoespasmo e, durante o tempo de oclusão, foram embebidos em solução salina fisiológica aquecida.

A contrastação dos vasa vasorum após a oclusão foi efetuada empregando-se uma associação dos métodos descritos por Vio e col.42 e por Wyatt e col. 44, com modificaçc̃es. O contraste utilizado foi a mistura de gelatina **: líquida a $5 \%$ e tinta da China *:* a 65\%, na proporção de 1:2, aquecida a aproximadamente $36 \circ \mathrm{C}$. A injeção manual do contraste foi realizada através do cateterismo retrógrado da artéria ilíaca comum direita, com catéter de polietileno com diâmetro externo de aproximadamente $1 \mathrm{~mm}$ (Venocath $18^{* * * * *}$ ), cuja extremidade distal foi localizada ao nivel da aorta ascendente. A quantidade de contraste injetada variou de 15 a $20 \mathrm{ml}$, de acordo con: o peso do animal. A observação visual da contrastação do focinho e da língua dc animal serviu de parâmetro para o término da injeção e normalmente o animal morre ao final da injeção ou minutos após o seu término. Previamente à injeção do contraste foi injetado, através do catéter, $1 \mathrm{ml}$ de solução de cloridrato de lidocaína a $1 \%$ para evitar vasoconstrição durante a contrastação.

Após a injeção de contraste os animais foram imersos $\epsilon m$ solução de formol a $10 \%$ por 24 horas, para a fixação inicial dos tecidos e endurecimento do contraste. Posteriormente os segmentos cervicais foram removidos em bloco e as artérias carótidas foram lavadas com solução salina físiológica, com a finalidade de remover o excesso de contraste retido na luz dos vasos. Após completar a fixação em solução de formol a $10 \%$ por 48 horas, as artérias foram dissecadas e removidas, incluindo 0 arco da aorta e o tronco braquiocefálico.

Os vasos removidos foram diafanizados, passando em uma bateria de álcoois e em xilol. A seguir foram observados sob lupa estereoscópica imersos em xilol e depois montados entre lâmina e lamínula com bálsamo do Canadá (preparação total) e observados e fotografados sob o microscópio óptico.

Quatro segmentos de artérias carótidas, representativos dos resultados observados ao exame direto, foram incluídos em parafina, segundo técnica rotineira, seccionados transversalmente (cortes de $10 \mathrm{u}$ ) e montados entre lâmina e lamínula para exame histológico an microscópio óptico. Os cortes foram observados apenas com o contraste

* Nembutal - Abbott Laboratórios do Brasil Ltda.

* Cloridrato de Lidocaina - Apsen Laboratórios S/A.

*** Gelatina em pó Otker - Cabeça Branca Produtos Alimentícios Ltda.

**** Tinta para desenho Rembrandt - IRCO Importadora Ltda.

**** Venocath 18 - Abbott Laboratories/North Chicago, U.S.A. 
proporcionado pela mistura injetada nos vasa vasorum e também corados pelo hemalumen. Foram estudados os seguintes segmentos: um proximal e outro distal ao local da clampagem de um animal do grupo 5; um segmento onde foi aplicado o microclampe e que se encontrava dilatado, de outro animal do grupo 5 e um segmento onde aplicado o microclampe, que apresentava um aglomerado de vasa vasorum (neovascularização), de um animal do grupo 9.

\section{RESULTADOS}

A. Observacão direta do material (preparações totais).

1 - Grupo 1 (controle) - quando a remoção do contraste da luz do vaso foi satisfatória, observamos por transparência as pregas longitudinais do endotélio, delineadas por filamentos de contraste retido no seu interior, dispostas paralelamente ao longo da luz do vaso (figura $1 \mathrm{~A}$ ).

A injeção de contraste da maneira descrita proporciona um enchimento adequado dos vasa vasorum de todo o sistema arterial. A nossa observação foi restrita às artérias carótidas comuns (figura 1A), em seus segmentos compreendidos entre as suas origens no arco aórtico e tronco braquiocefálico, até a bifurcação em carótidas externa e interna.

Mediante a técnica microcirúrgica empregada, não observamus diferenças entre os segmentos das artérias dissecadas (carótidas direitas), quando comparadas com os segmentos correspondentes das artérias não dissecadas (carótidas esquerdas).

Os vasa vasorum que acompanham as artérias carótidas constituem uma densa rede formada por ramos de calibre variável, localizada principalmente na adventícia destes vasos, que se continua a partir do arco da aorta e recebe ramos adicionais provenientes de pequenos ramos das artérias tireóideas.

Esta rede pode ser esquematizada, simplificadamente, da maneira que segue:

a) Grandes troncos (figura 1B) - apresentam-se em número variável e dispõe-se na adventícia das carótidas, paralelamente ao eixo longitudinal do vaso. Em algumas preparacões conseguimos distinguir ramos arteriais e ramos venosos entre os grandes troncos, baseado nas características descritas por Gozzetti \& Vio 16.

Os troncos arteriais apresentam-se em menor número, têm menor calibre e contornos mais regulares e apresentam ramificações que lhe são oblíquas ou perpendiculares (figura 1B). Os ramos primários dos vasa vascrum arteriais subdividem-se várias vezes na adventícia das carótidas e vão terminar em uma rede capilar muito fina (fjgura $1 D$ ).

Os troncos venosos são mais calibrosos, têm contornos mais irregulares e geralmente mais de um destes troncos acompanha um tronco arterial (figura 1B). Eles recebem as confluências de ramos menores provenientes dè uma rede grosseira. Os ramos que desembocam nos troncos venosos unem-se a eles formando ângulos retos ou agudos e às vezes são praticamente paralelos aos troncos. Muitas vezes os grandes troncos apresentam comunicacões entre si $€$ ocasionalmente estas comunicaçôes ocorrem entre troncos distantes. dando ao conjunto um aspecto plexiforme. 


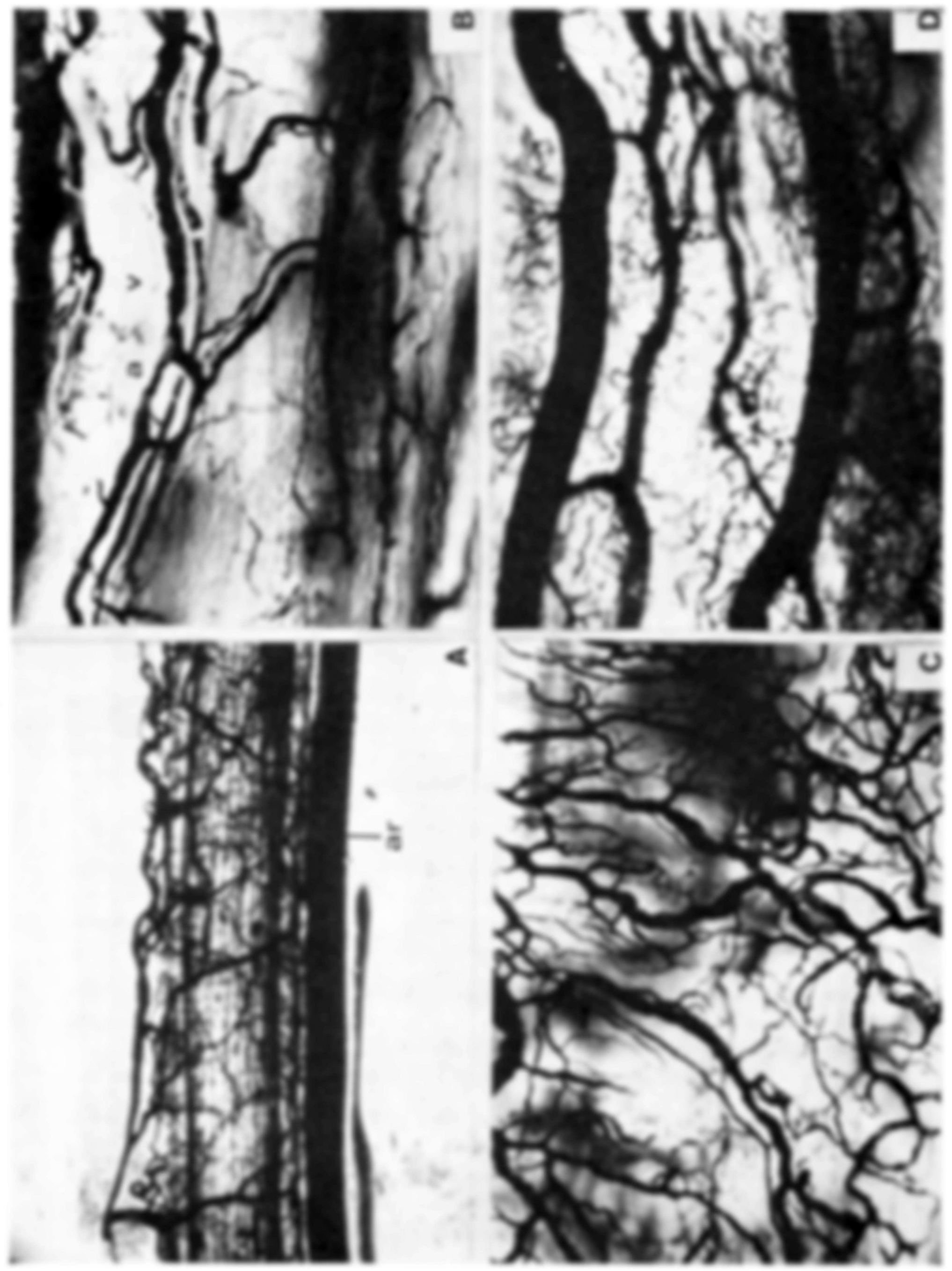


Fig. 1 - Fotomicrografias de segmentos de artérias carótidas comuns normais com seus vasa vasorum contrastados. $\operatorname{Em} A(15 X)$, Visão panoramica: ar = artefato provocado pela camada de bálsamo. Observar o pregueamento longitudinal do endotélio; em $B(81,2 X)$, em $C(38,4 X)$ e em $D(89 X)$, detalhes da rede de vasa vasorum mostrando os grandes troncos arteriais (a) e venosos (v), anastomoses entre os grandes troncos, rede de malhas grosseiras (C) e rede capilar de malhas finas (D).

b) Rede de malhas grosseiras (figura 1B) - observanıos na adventicia das carótidas uma rede de vasa vasorum, de malhas grossas e irregulares, constituída em sua maioria por ramos venosos que vão confluindo e formando ramos maiores, até originarem os afluentes dos grandes troncos venosos. Outros componentes desta rede sâo os ramos arteriais resultantes das subdivisões sucessivas dos ramos primários dos troncos arteriais. Embora frequentemente tivéssemos observado ramos arteriais qua se comunicavam diretamente com ramos venoscs resta rede, năo os conseguimos documentar, pois as preparações totais não permitiam profundidade de foco.

c) Rede capilar de malhas finas (figura 1D) - em toda a adventicia das carótidas observamos uma intrincada rede de finos vasos, com malhas poligonais, que parece representar uma rede capilar entre os ramos arteriais terminais e a rede venosa grosseira.

2 - Grupos 2 a 7 (animais com sacrificio precoce) - a artéria carótida submetida à clampagem tenıporária permaneceu pérvia em todos os animais estudados, fato constatado pela excelente contrastação do sistema arterial distal ao local da clampagem.

Nos animais dos grupos 2 e 3 o local da clampagem estava marcado por uma dilatação discreta, por um «embaçamento» discreto da parede vascular e/ou retenção de contraste no endotélio. Em apenas 2 animais destes grupos o local onde o microclampe foi aplicado não apresentou alterações que permitissem sua identificação.

Nos animais dos grupos 4 a 7 o local da clampagem estava marcado por uma ou por uma combinação das seguintes alteraçôes morfológicas: retenção de contraste no endotélio (figuras 2A e 2B), «embaçamento» da parede vascular, dilatação (figuras 2A e 2B) e vestígio de hemorragia perivascular (presença de uma coloração amarelada na parede do vaso, resultante da degradação da hemoglobina liberada nor hemácias destruidas).

As pregas longitudinais do endotélio foram observadas no local da clampagem em vários animais destes grupos e estavam alteradas (diminuídas, desorganizadas ou alargadas) na maioria deles (figuras 2A e 2B). Em alguns animais estas pregas não foram observadas devido ao excesso de contraste na luz do vaso.

Os grandes troncos dos vasa vasorum apresentaram-se em menor número no local da clampagem (figuras (figuras $2 A$ e 2B) na maioria dos animais dos grupos 2 a 7. Em alguns casos, além de diminuídos em número, os grandes troncos estavam mal contrastados e com irregularidades nas paredes.

A rede de malhas grosseiras contrastou-se mal ou não se contrastou no local da clampagem na maioria dos animais dos grupos 2 a 7. Enı alguns animais esta rede 


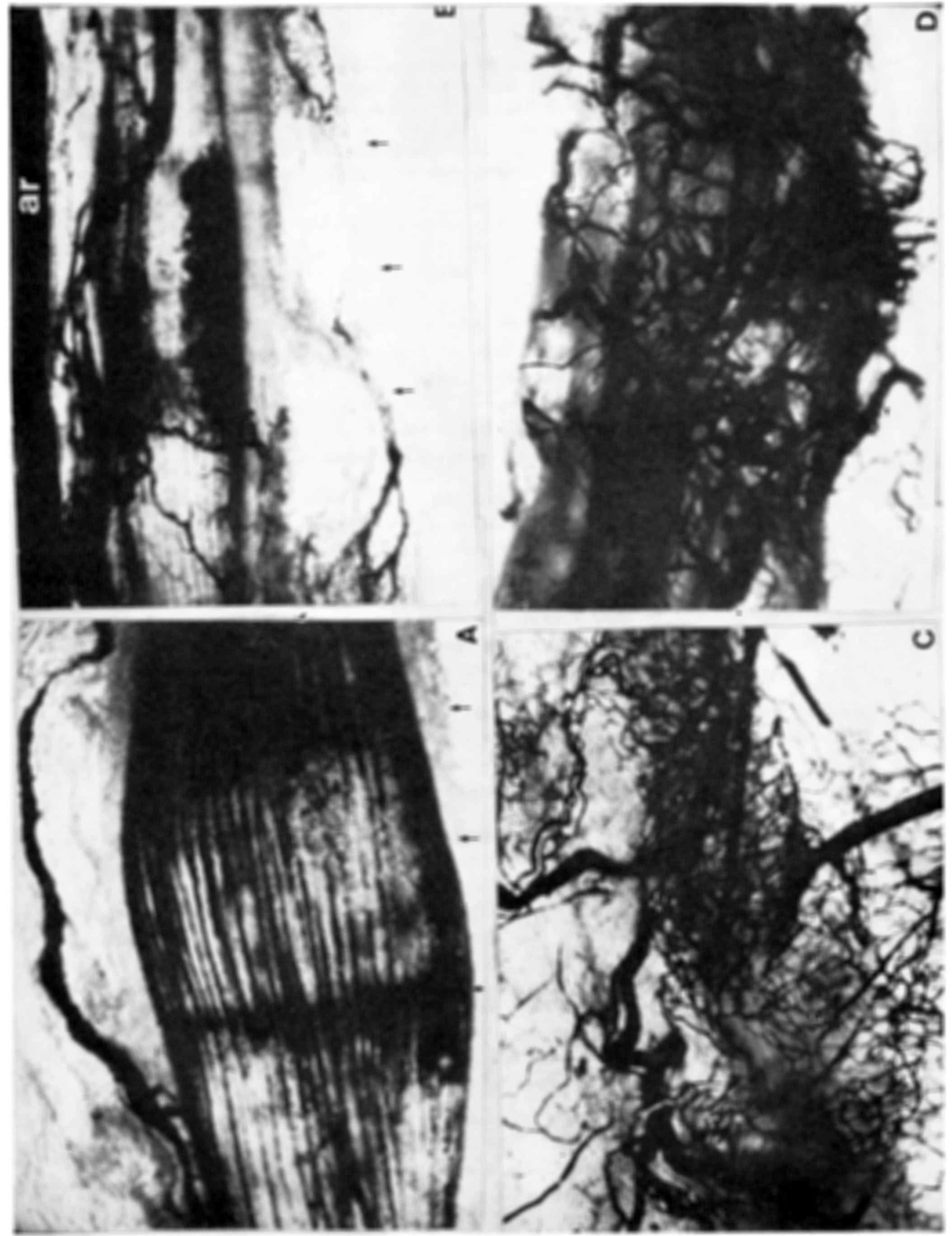


Fig. $2-A, B$ e $C$, fotomicrografias de segmentos de artérias carotidas comuns com seus vasa vasorüm contrastados apos 10 minutos de clampagem com microclampe de Yasargil. $E m A$ $B$ (41X), presença de dilatação, retenção de contraste no endotélio, diminuição do número de grandes troncos $e$ ausência da rede grosseira no local da clampagem (setas). Observar as alteracóes das pregas longitudinais do endotélio no local da clampagem e próximo a ele; $a r=$ artefato provocado pela camada de bálsamo. Em $C,(84,8 X)$, rede capilar de malhas finas ectasica distalmente ao local da clampagem (comparar com a figura 1C). Em $D$, (110X), segmento da artéria carótida comum com seus vasa vasorum contrastados 30 dias apos a clampagem por 30 minutos com microclampe de Biemer, mostrando um aglomerado de vasa vasorum no local da clampagem.

estava, além de mal contrastada, com as malhas desorganizadas e com os espaços aumentados.

A rede capilar de malhas finas contrastou-se mal ou não se contrastou no local da clampagem em todos os animais dos grupos 2 a 7 . Nos animais em que esta rede contrastou-se mal, observava-se apenas uma «sombra» desta rede.

Distalmente ao local da aplicação do microclampe foram observadas ectasias dos grandes troncos dos vasa vasorum e da rede de malhas grosseiras na maioria dos animais dos grupos 2 a 7 e, da rede capilar de malhas finas (figura 2C), em alguns animais destes grupos.

3 - Grupos 8, 9 e 10 (animais com sacrificio tardio) - o local da clampagem pode ser identificado na maioria dos animais destes grupos por uma ou por uma combinação das seguintes alterações morfológicas: retenção de contraste no endotélio, dilatação, «embaçamento» da parede e/ou um aglomerado de vasa vasorum sinuosos (neovascularização), constituído por grandes tronccs, seus ramos principais e pela rede de malhas grosseiras (figura 2D).

As pregas longitudinais do endotélio foram observadas em vários animais destes grupos e apresentaram-se alteradas (diminuídas, desorganizadas, alargadas ou ausentes) em todos eles. Em alguns animais estas pregas não foram observadas devido à neoformacão de vasa vasorum no local da clampagem, que impedia a sua visualização.

Os grandes troncos dos vasa vasorum e a rede de malhas grosseiras apresentaram-se ectásicos no local da clampagem na maioria dos animais destes grupos.

A rede capilar de malhas finas apresentou-se ora mal contrastada ora ectásica, no local da clampagem, em todos os animais destes grupos e geralmente com malhas irregulares e espacos aumentados.

Distalmente ao local da aplicação do microclampe foram observadas ectasias dus grandes troncos dos vasa vasorum e da rede de malhas grosseiras na maioria dos animais destes grupos e da rede capilar de malhas finas em apenas alguns animais.

\section{B, Exames histologicos}

Os cortes transversais da artéria carótida comum de um animal do grupo 5, proximais ao local da clampagem, mostraram a presença da rede de vasa vasorum adventicial 

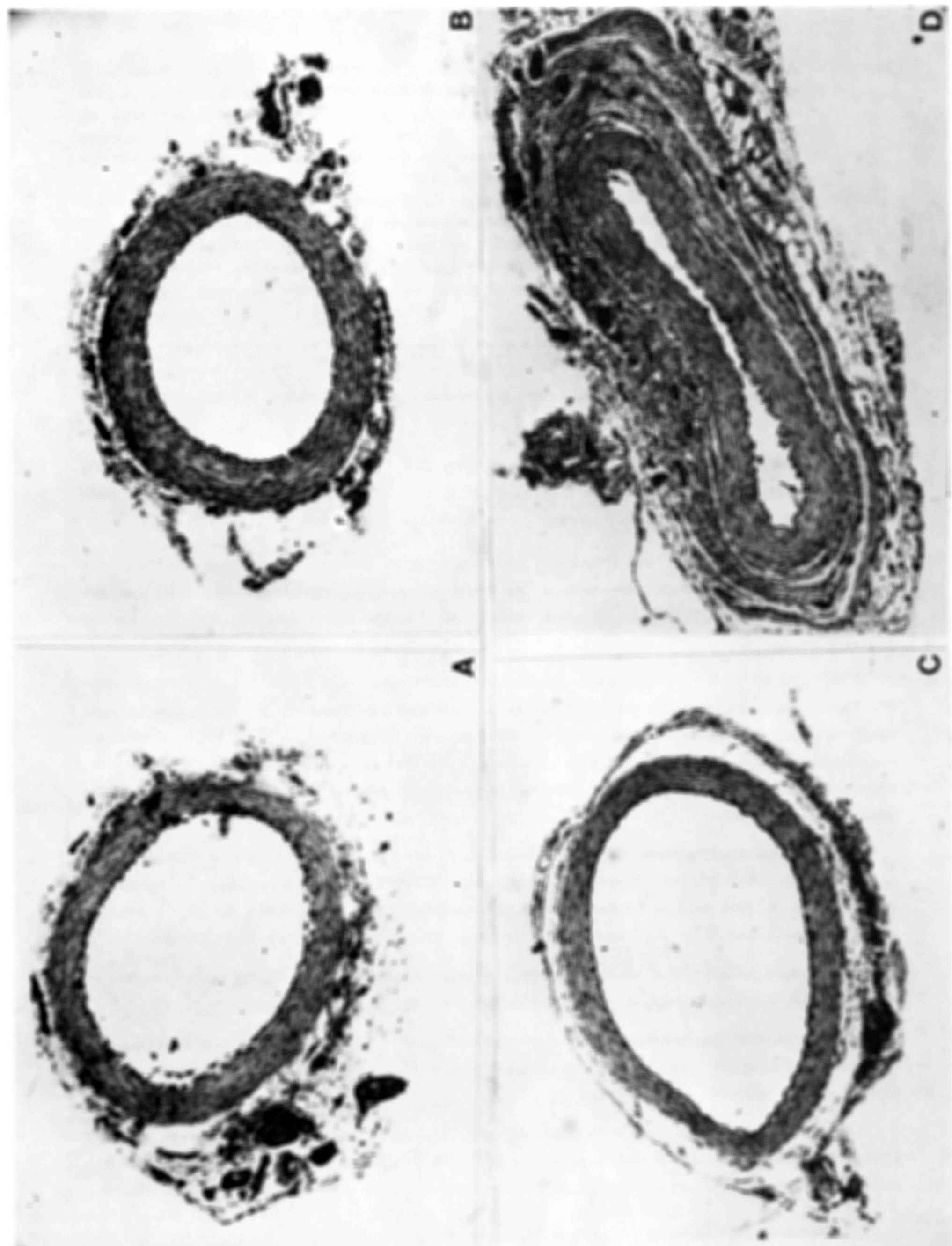
Fig. 3 - Cortes transversais de segmentos de artérias carotidas comuns submetidas a clampagem temporária por 30 minutos com microclampe de Yasargil (A, $B$ e $C$ - sacrificio precoce; $D$ - sacrificio tardio), com os vasa vasorum contrastados com gelatina e tinta da China. Em $A, B$ e $C$ (170X), cortes proximal (A), no local da clampagem (B), que se enoontrava dilatado, $e$ distal ao local da clampagem (C). Observar o pregueamento normal do endotélio em $A$ e $C$ e irregular e pouco acentuado em $B$; $a$ distribuicão dos vasa vasorum apenas na metade da circunferencia do vaso em $B$ e a ectasia dos vasa vasorum em $C$. Em $D,(140 X)$, corte ao nivel do local da clampagem (onde ocorreu um aglomerado de vasa vasorum). Observar o pregueamento irregular e pouco acentuado do endotélio, o espessamento da adventicia com aumento da quantidade e ectásia dos vasa vasorum, o adelgacamento da camada média $e$ a descontinuidade do endotélio (setas).

ocupando toda a circunferência da artéria, embora com predominio en uma área determinada (figura 3A). Os cortes de áreas deste mesmo vaso, distais ao local da clampagem, mostraram distribuição semelhante dos vasa vasorum, mas estes encontravam-se ectásicos (figura 3B). O endotélio vascular apresentou um pregueamento nitido e regular, tanto nas áreas proximais como nas distais ao local da clampagem (figuras 3A e 3B), correspondendo às pregas longitudinais observadas no endotélio dos vasos nas preparações totais (vide figura 2A).

Os cortes transversais da artéria carótida comum de outro animal do grupo 5 , realizados no local da clampagem, onde havia uma dilatação evidente, mostraram um adelgaçamento da camada média e também os vasa vasorum posicionados apenas na metade da circunferência da artéria (figura $3 \mathrm{C}$ ). O pregueamento do ehdotélio estava irregular e menos nítido nestes cortes (figura $3 \mathrm{C}$ ).

Os cortes transversais da artéria carótida comum de um animal do grupo 9, realizados no local da clampagem, onde havia um aglomerado de vasa vasorum, mostraram um espessamento da adventícia possivelmente representando uma reação reparadora, com aumento do número de vasa vasorum e um adelgaçamento da camada média da parede arterial (figura 3D). O pregueamento do endotélio estava nitidanente alterado nestes cortes, apresentando-se irregular e pouco acentuado (frouxo), inclusive com descontinuidade em alguns pontos (figura 3D).

\section{COMENTARIOS}

As alterações agudas (animais sacrificados precocemente), observadas na parede das artérias carótidas da cobaia, submetidas à clampagem temporária, caracterizaram-se por lesões em todas as camadas do vaso, no local onde foi aplicado o microclampe.

A observação panorâmica dos vasos clampados, sob o microscópio óptico, mostrou que o local onde o microclampe foi aplicado pode ser identificado facilmente na maioria dos casos. Essa identificação foi possível pela presença de alterações das pregas longitudinais do endotélio, por retenção de contraste no endotélio, por dilatação local, por "embaçamento" da parede do vaso ou pela presença de hemorragia perivascular. 
As alterações das pregas longitudinais do endotélio representam lesões iniciais da parede, quando observadas ao microscópio eletrônico 12,22,32,41 e resultam da lesão da lâmina elástica interna 41 .

A retenção de contraste no endotélio ocorre devido à penetração do material nas áreas onde houve ruptura do endotélio com exposição das camadas subjacentes e representa um grau mais avançado de lesão da parede vascular, conforme observado ao microscópio eletrônico por vários autores 12,22,32,41.

O "embaçamento" da parede vascular observado no local da clampagem após a. sua diafanização é devido provavelmente ao esmagamento das fibras musculares da camada média, que prejudica a diafanização neste local. Esta alteração traduz portanto uma lesão grave da parede arterial.

A dilatação observada no local da aplicação do microclampe foi verificada por outros autores $12,22,32,41$ e é atribuída à necrose da média e da lâmina elástica interna 32 .

A presença de hemácias na camada média de artérias nos locais de aplicação de microclampes, foi relatada por Dujovny e col.12, que atribuem o fato à separação das fibras musculares por ação do microclampe. A observação de vestígios de hemorragia perivascular (verificada ao microscópio óptico por coloração amarelada ao redor do vaso), em alguns dos nossos casos, sugere que tenha ocorrido diapedese de células do sangue circulante na luz do vaso, devida às graves lesões da sua parede, uma vez que não observamos sangramento durante a dissecção e a clampagem.

A intensidade das lesões da parede de pequenos vasos submetidos à aplicação de microclampes varia de acordo com a pressão exercida pelas suas lâminas sobre os vasos e com o tempo de duração da clampagem. A pressão exercida pelas lâminas é decorrente das características físicas dos microclampes 10 e é proporcional ao momento de alavanca compreendido entre o ponto que entra em contato com a parede do vaso e o fulcro do microclampe 12,39.

Outro fator que teoricamente poderia influenciar a intensidade da lesão da parede vascular é o diâmetro do vaso clampado. Entretanto não acreditamos que este fator interfira com os nossos achados. Richling e col. 32 mostraram que não existe correlação entre o grau de lesão e o diâmetro do vaso clampado, para vasos com diâmetros de até $2 \mathrm{~mm}$ (limite superior), faixa de variação dentro da qual situam-se as artérias por nós observadas.

As lesões da parede vascular observadas ao microscópio eletrônico iniciam-se precocemente e independem do tempo de duração da clampagem quando a força exercida pelas lâminas do microfilme sobre a parede do vaso é alta (maior que 50-60 g) 15,32.41. Para os microclampes que exercem pressões menores a intensidade das lesões é proporcional ao tempo de clampagem 32. Sugita e col. 35 observaram, ao microscópio óptico, lesões precoces da parede vascular (rupturas da membrana elástica interna e de fibras musculares) apenas com microclampes que exercem pressões acima de $120 \mathrm{~g}$. Não observaram ruptura da intima em nenhuma das situações. 
Os nossos resultados são compativeis com as observações à microscopia eletrônica realizadas por alguns autores ${ }^{15.32 .41}$ quanto ao aparecimento das lesões com pequena duração da clampagem (10 minutos), utilizando microclampes com forças de abertura de $51 \mathrm{~g}$ (Biemer), $148 \mathrm{~g}$ (Yasargil) e $274 \mathrm{~g}$ (Medizin), e contrariam as observações de Sugita e col. 39. Considerando-se o mesmo tipo de microclampe, a clampagem dos vasos durante 30 minutos não acrescentou novos tipos de lesões nem provocou lesões mais graves na parede vascular, confirmando as observações de Thurston e col. 41.

As lesões agudas da parede vascular foram menos frequentes e menos graves nos vasos submetidos ao microclampe de Biemer pois neles o local da clampágem pode ser identificado ao microscópio óptico por lesões mais evidentes na intima (alterações das pregas longitudinais do endotélio e retenção de contraste) e pouco evidentes na camada média ("embaçamento" e dilatação local discretos da parede vascular). Para os outros microclampes (Yasargil e Medizin), o local da clampagem pode ser identificado por lesões da íntima e a ocorrência de lesões das outras camadas da parede ("embaçamento" da parede, dilatação local e vestígio de hemorragia perivascular), foi a regra.

Dujovny e col. 13 mostraram que quando se aplica a força mínima de oclusão vascular sobre a parede de um vaso, através de um microclampe $(6,9$ a 16,1 g para vasos com diâmetros de 1,8 a $2,4 \mathrm{~mm}$ ), o grau de lesão da intima é mínimo. Mostraram também que existe uma relação direta entre 0 acréscimo de força sobre a parede e o grau de lesão da intima.

Não observamos dif'erenças entre as lesões provocadas pelos microclampes de Yasargil e Medizin, apesar da grande diferença entre as suas forças de abertura (148 e $274 \mathrm{~g}$ respectivamente). Este fato sugere que existe uma determinada pressão exercida pelas lâminas do microclampe que ocasiona $o$ grau máximo de lesão da parede vascular. Acima desta pressão deixa de existir a relação direta entre o aumento da pressão e o grau de lesão da parede, proposta por Dujovny e col. 13.

As alteraçoes agudas observadas na adventícia dos vasos clampados temporariamente foram essencialmente as dos vasa vasorum.

A contrastação do sistema vascular da cobaia pela metodologia descrita proporcionou boa visualização dos vasa vasorum das artérias carótidas.

Apesar das características morfológicas da rede de vasa vasorum representarem apenas o momento funcional da parede do vaso 18,26 , a sua observação permitiu uma avaliação muito boa das alterações que ocorreram nos locais onde os microclampes foram aplicados, devido à possibilidade de efetuarmos uma comparação com o modelo experimental estabelecido no grupo controle. Ademais, a utilização da técnica microcirúrgica na dissecção dos vasos, possibilitou o isolamento adequado da área a ser clampada, conforme as observaçōes efetuadas no grupo controle, sem prejuízo da rede de vasa vasorum.

Este fato é de grande importância pois, com a metodologia de contrastação utilizada, a lesão dos grandes troncos dos vasa vasorum da adventícia provo- 
caria um extravasamento do contraste ao redor do vaso, prejudicando o enchimento adequado e a visualização de toda a rede de vasa vasorum, principalmente os ramos de menor calibre. A ausência de contrastação e a desorganização da rede de vasa vasorum adventicial, observadas em casos de dissecção sem técnica microcirúrgica, indica que o enchimento desta rede se faz fundamentalmente através de ramos provenientes da própria artéria ou do tecido periadventicial e não por difusão do contraste a partir da luz do vaso.

No local da aplicação do microclampe observamos uma diminuição do número dos grandes troncos dos vasa vasorum, diminuição ou mesmo ausência da rede de malhas grosseiras e da rede capilar, além de irregularidades na contrastação destes vasos.

Não foi possível estabelecer diferenças entre as alterações apresentadas pelos vasa vasorum no local da aplicação para cada tipo de microclampe e também quanto à duração da clampagem (10 ou 30 minutos), pois todos os tipos e intensidades de lesões foram observados precoce e independentemente do tipo de microclampe utilizado, confirmando em parte as observações de Marzoli e col. 26. Estes autores observaram que o quadro morfológico dos vasa vasorum atinge o estado extremo de alterações à mais leve modificação da estrutura da parede do vaso.

Nas regiōes distais ao local da clampagem, toda a rede de vasa vasorum, mas principalmente os grandes troncos e a rede de malhas grosseiras, apresentava-se ectásica, provavelmente um fenômeno vicariante na tentativa de melhorar o suprimento sanguíneo da parede submetida à hipóxia temporária pela ação do microclampe.

Thurston e col. 41 e Dujovny e col. 12 mostraram, ao microscópio eletrônico, que as alterações agudas observadas na intima de artérias submetidas à clampagem temporária recuperam-se parcialmente após alguns dias, ocorrendo a regeneração do endotélio que, entretanto, nunca volta ao normal. A persistência da dilatação do vaso no local da aplicação do microclampe foi observada por Thurston e col. 41, após 7 dias da clampagem. Os autores sugerem que a necrose da camada média, que leva à formação da dilatação, incapacitaria o vaso a contrair-se em resposta aos processos fisiológicos normais, constituindo-se enı um importnte fator na ocorrência de trombose arterial tardia inexplicada, que se verifica após um episódio de trauma ou vasoconstrição.

Em nossas observações, as alterações crônicas (animais sacrificados após 30 dias), verificadas nas paredes das artérias clampadas temporariamente evidenciaram recuperação parcial em relação àquelas lesões agudas verificadas nas artérias dos animais sacrificados precocemente.

Esta recuperação foi mais evidente nos vasos submetidos ao microclampe de menor força (Biemer) pois neles não observamos alterações sugestivas de lesões da intima ou da camada média na maioria dos animais. Nos vasos submetidos aos microclampes de Yasargil e Medizin, o local da aplicação do microclampe ainda apresentava alterações sugestivas de lesões da intima (alte- 
raçōes das pregas longitudinais e retenção de contraste no endotélio) e da camada média ("embaçamento" da parede, dilatação local e adelgaçamento da camada média). Estas observações mostram a existência de uma correlação inversa entre a pressão exercida pelo microclampe e a recuperação das lesões da parede vascular e também sugerem, da mesma forma que para as lesōes agudas, a existência de uma pressão crítica, que determina o grau máximo de lesão da parede vascular, cuja recuperação ocorre sempre com maiores sequelas.

As alterações crônicas apresentadas pelos vasa vasorum no local da aplicação do microclampe caracterizaram-se pela presença de neovascularização (com vasos provenientes da adventícia e da região periadventicial circunvizinha), acompanhada de um espessamento local da adventícia. $O$ quadro observado demonstra uma reaçăo reparadora relativamente desordenada, equiparando-se a um processo cicatricial. Não foram observadas diferenças neste quadro em relação ao tipo de microclampe utilizado.

Estas alterações foram semelhantes às observadas por certos autores em outros tipos de agressão à parede vascular, como a provocação de estenose temporária de grau variado 16 , adventiciectomias $9,18,33$, suturas de arteriotomias 43 e a reparação de defeitos arteriais com enxertos ou próteses $17,19,24,25,27,28,40,44$.

As alterações observadas em todas as camadas da parede de pequenas artérias, nos locais onde foi aplicado o microclampe, podem ocorrer por dois mecanismos: diretamente pelo trauma mecânico causado pela pressão exercida pelas lâminas do microclampe ou indiretamente, pelo comprometimento da circulação da parede arterial.

As lesões diretas determinadas pela pressão exercida pelas lâminas é traduzida pelo esmagamento imediato das células e a estrutura mais vulnerável da parede parece ser a intima. Provavelmente existe uma relação direta entre o grau de lesão pelo trauma mecânico e a pressão exercida pelo microclampe, independente do tempo de duração da clampagem. Com a aplicação de pressões maiores as outras camadas da parede também são comprometidas.

As lesões determinadas por isquemia da parede arterial podem resultar em necrose celular em qualquer camada da parede e dependem fundamentalmente do tempo de duração da clampagem, sendo portanto muitc importantes nas alterações determinadas por microclampes que exercem pequenas pressões sobre a parede do vaso. Anatomicamente a camada média é a região da parede arterial mais suscetivel às lesões isquêmicas, pois é a zona de transição entre os dois tipos de irrigação da parede arterial (difusão do sangue circulante e rede de vasa vasorum). Por esse motivo ela é a região que maior dificuldade apresenta no processo de recuperação das lesões pós-clampagem temporária, persistindo o seu adelgaçamento como sequela.

Não observamos correlação entre o grau de lesão da parede arterial e as alterações apresentadas pela rede de vasa vasorum, conforme referido por Schlichter 33. Este fato deve ser atribuido à possibilidade de ser mais impor- 
tante o mecanismo de lesão direta da parede de pequenas artérias pelos microclampes e também porque, na nutrição arterial, quanto menos espessa é a parede, maior é a importância da difusão do sangue circulante através da sua parede, em detrimento da irrigação pela rede de vasa vasorum 18,26.

A questão da importância que poderiam ter estas graves alterações observadas nas paredes de pequenas artérias submetidas à clampagem temporária, em situações clínicas reais, já foi formulada por Acland \& Trachtenberg 2, com relação às lesões provocadas por anastomoses microvasculares experimentais.

Do ponto de vista essencialmente funcional temos que considerar que as alterações observadas não provocaram trombose com oclusão arterial em nenhum dos animais estudados, que é o problema mais sério levantado pelos autores que se dedicam à microcirurgia vascular 1,2,3,12,15,20,22,30,31,32,37,41. Este fato é um argumento importante a favor da inocuidade destes procedimentos a longo prazo, constatação feita anteriormente por Acland \& Trachtenberg 2.

Persiste ainda a interrogação de como responderia o segmento lesado destes pequenos vasos a regimes de hipertensão arterial, patologia bastante comum em nossos dias. Tudo nos leva a crer que esta área seria uma região propensa ao desenvolvimento de um aneurisma.

Considerando-se que as cirurgias intracranianas em que os microclampes temporárias são utilizados com relativa frequência são realizadas em pacientes que apresentam problemas na árvore circulatória, teremos uma somação de fatores semelhantes àquela que ocorre na patogênese dos aneurismas saculares intracranianos 23. Nesta, ao substrato anatômico congênito da parede arterial (defeitos na membrana elástica interna e na camada média) juntam-se os fatores adquiridos (aterosclerose e hipertensão arterial), com todos os riscos inerentes à ruptura de um aneurisma intracraniano.

Para a confirmação desta possibilidade ainda são necessários estudos experimentais das propriedades físicas da parede de pequenos vasos submetidos à clampagem temporária e estudos histológicos de casos fortuitos de necrópsia em que os pacientes tenham sido submetidos anteriormente a tais procedimentos cirúrgicos

\section{CONCLUSOEES}

O estudo das artérias carótidas da cobaia (Cavia porcellus), submetidas à clampagem temporária (10 e 30 minutos) com microclamples de Biemer, Yasargil e Medizin e cujos vasa vasorum foram contrastados pela técnica descrita, permitiu as seguintes conclusões:

1. Quando as preparações técnicas foram satisfatórias pode-se demonstrar que a oclusão temporária provocou lesões agudas em todas as camadas da parede arterial.

2. As lesões agudas observadas no local da clampagem ocorreram independentemente da duração da oclusão. 
3. Considerando-se as lesões provocadas pelos três tipos de microclampes utilizados podemos dizer que existe uma pressão crítica exercida pelas lâminas de um microclampe sobre a parede de um vaso, acima da qual deixa de existir uma relação direta entre a pressão exercida e o grau de lesão da parede vascular.

4. Não foram observadas diferenças entre os tipos de microclampes utilizados especificamente para as alterações da rede de vasa vasorum adventicial.

5. A observação das artérias 30 dias após a oclusão evidenciou recuperação parcial em relação às lesões agudas da parede arterial, especialmente para o microclampe de Biemer, exceto para as lesões dos vasa vasorum.

6. Apesar desta recuperação, persistiram lesões na intima e na camada média no local da clampagem que poderão constituir pontos potencialmente fracos na parede do vaso.

7. Não foram observadas diferenças nos quadros morfológicos reacionais apresentados tardiamente pelos vasa vasorum, em relação ao tipo de microclampe utilizado.

8. Não houve correlação entre o grau de lesão da parede arterial e as alterações dos vasa vasorum. O trauma mecânico direto pareceu ser mais importante que a isquemia na gênese destas lesões.

9. Apesar das lesões graves, agudas e crônicas, observadas nas artérias clampadas temporariamente, não foram observadas trombose e oclusão arterial no local da clampagem, 30 dias após a oclusão temporária.

\section{RESUMO}

Foram estudadas as lesões provocadas nas artérias carótidas de cobaias (Cavia porcellusi), com diâmetros variando de 1 a $2 \mathrm{~mm}$, submetidas à clampagem temporária (10 e 30 minutos), com microclampes empregados em microneurocirurgia, utilizando-se injeção intravital da mistura de gelatina líquida e tinta da China para contrastação dos vasa vasorum. As artérias foram diafanizadas e observadas ao microscópio óptico e posteriormente observadas através de cortes histológicos.

Foram verificadas lesões agudas na íntima, na camada média e na rede de vasa vasorum adventicial, que ocorreram independentemente da duração da clampagem. Os resultados sugerem que existe uma pressão crítica exercida pelos microclampes, acima da qual deixa de haver uma relação direta entre a pressão exercida e o grau de lesão da parede arterial.

A observação das artérias 30 dias após a clampagem temporária evidenciou recuperação parcial em relação às lesões agudas embora tenham persistido alterações na íntima e na camada média, que poderão constituir pontos fracos na parede arterial. 
As alterações graves dos vasa vasorum observadas agudamente e o quadro morfológico reacional verificado tardiamente não apresentaram diferenças quanto à pressão exercida pelo microclampe.

Não foi possível correlacionar o grau de lesão da parede arterial e as alterações dos vasa vasorum. O trauma mecânico provocado pelas lâminas do microclampe pareceu mais importante na gênese destas lesões.

Apesar das lesões graves observadas na parede das artérias clampadas temporariamente, em nenhum caso verificou-se trombose e oclusão vascular no local da clampagem.

\section{SUMMARY}

Lesions of carotid artery wall in the Guinea pig after temporary clipping with special attention to the vasa vasorum network.

The author analyses the lesions of carotid artery wall in the Guinea pig (Cavia porcellus) submitted to temporary clipping with clips used in microvascular neurosurgery, by means of intravital injection of gelatin and India ink to contrast the vasa vasorum. The arteries were cleared with xilol and observed on the optic microscope and later throught histologic sections.

Acute lesions without relationship to the clipping time were verified in the intima, tunica media and in the vasa vasorum network of the adventitia. The results suggest that there is a critical clip pressure, above that there is no direct relationship between the clip pressure and the severity of the lesion of the arterial wall.

The arteries observed 30 days after the temporary clipping showed a partial recuperation relative to the acute lesions, although alterations in the intima and tunica media persisted and will constitute weak points in the arterial wall.

Differences relative to the clip pressure were not observed in the acute severe alterations of the vasa vasorum and in the later reactional picture showed by these vessels.

It was not possible correlate the severity of the lesions of the arterial wall and the alterations of the vasa vasorum. It is believed that the mechanical trauma due to clip pressure was more important than the ischemia in the genesis of these lesions.

Despite the severity of the lesions observed in the arterial wall submitted to temporary clipping, thrombosis and vascular occlusion were not observed in the studied animals.

\section{REFERENCIAS}

1. ACLAND, R. D. - Thrombus formation in microvascular surgery: An experimental study of the effects of surgical trauma. Surgery 31:766, 1973.

2. ACLAND, R. D. \& TRACHTENBERG, L. - The histopathology of small arteries following experimental microvascular anastomosis. Plast. Reconstr. Surg. 59:868, 1977. 
3. BAXTER, T. J.; O'BRIEN, B. M.; HENDERSON, P. N. \& BENNET'T, R. C. The histopathology of small vessels following microvascular repair. Brit. J. Surg. $59: 617,1972$.

4. BENJAMIN, H. B., BARTENBACK, G. \& ZEIT, W. - The importance of the vasa vasorum of aorta. Surg. Gynecol. Obst. 110:224, 1960.

5. BROOK, W. H.; BAIRD, R. J. \& SILVER, M. D. - Vasa vasorum in autogenous dog vein by-pass grafts. J. Cardiovasc. Surg. (Torino) 15:655, 1974.

6. CLARKE, J. A. - An x-ray microscopic study of the vasa vasorum of the normal human ascending aorta. British Heart J. 27:99, 1965.

7. CLARK, J. A. - An x-ray microscopic study of the normal root of neck arteries in man. Thorax 20:270, 1965.

8. ClARKE, J. A. - An x-ray microscopic study of the vasa vasorum of the intracranial arteries. Ztschr. f. Anat. Ent. 124:396, 1965.

9. DAHL, E. V.: EDWARDS, E. C.; GRINDLAY, J. H. \& EDWARDS, J. E. - Effect of removal of adventitia from arteries: an experimental study. Surgery 50:533, 1961.

10. DELONG, W. B. \& RAY, R. L. - Metallurgical analysis of aneurysm and microvascular clips. J. Neurosurg. 48:614, 1978.

11. DE SOUZA, A. \& ALVARES, L. - Microangiographic studies of the vasa vasorum of the thoracic anrta. Radiolngy $75: 91,1960$.

12. DUJOVNY, M.; OSGOOD, C. P.; BARRIONUEVO, P. J.; PERLIN, A. \& KOSSOVSKI, N. - SEM evaluation of endothelial damage following temporary middle cerebral artery occlusion in dogs. J. Neurosurg. 48:42, 1978.

13. DUJOVNY, M.; WAKENHUT, N.; KOSSOVSWI, N.; GOMES, C. W.; LAHA, R. K. ; LEFF, L. \& NELSON, D. - Minimum vascular occlusive force. J. Neurosurg. $51: 662,1979$.

14. ERDHEIM, J. - Medionecrosis aortae idiopathica cystica. Virch. Arch. f. Path. Anat. 276:187, 1930.

15. GERTZ, S. D.; RENNELS, M. L.; FORBES, M. S.; KAWAMURA, J.; SUNAGA, T. \& NELSON, E. - Endothelial cell damage by temporary arterial occlusion with surgical clips: study of the clip site by scanning and transmission electron microscopy. J. Neurosurg. 45:514, 1976.

16. GOZZETTI, G. \& VIO, A. - Considerazioni su alcuni aspetti morfologici della rete capillare di vasa vasorum in condizioni normali e patologiche indotte sperimentalmente. Boll. Soc. Ital. Biol. Sper. 41:980, 1965.

17. GOZZETTI, G.; PLATANL1, A.; IrEGGIANI, A. \& VIO, A. - Morphological and functional alterations of the vasa vasorum following the repair of arterial defects by means of prosthesic patch. Experimental research on the thoracic aorta of dogs. Angiologica 2:182, 1965.

18. GOZZETTI, G.; VIO, A. \& REGGIANI, A. - Comportamento dei vasa vasorum. dopo avventiziectomia arteriosa nel cane. Boll. Soc. Ital. Biol. Sper. 43:85, 1967.

19. HALPERT, B.: O'NEAL, R. M.: JORDAN JR., G. L. \& DE BAKAY, M. E. Vasa vasorum of dacron prosthesis in canine aorta. Arch. Path. 81:412. 1966.

20. HARASHIMA, T. - The site of reapplication of microvascular clamps. Plast. Reconstr. Surg. 58:719, 1976.

21. HUEPER, W. C. \& ICHNIOWSKI, C. T. - Experimental studies in cardiovascular pathology: late vascular reactions of histamine shock in dogs. Am. J. Pathol. 20:211, 1944.

22. LECLERC, P.; GUIDOIN, R. \& HEBRT, G. - tude du traumatisme causé a l'endothélium par le rapprocheur de clamp e les clamps utilisés en microchirurgie vasculaire. Union Med. Can. 106:1513, 1977.

23. LOUGHEED, W. M. \& BARNETT, H. J. M. - Lesions producing spontaneous hemorrhage. In YOUMANS, J. R. - Neurological surgery. W. B. Saunders Company, Philadelphia 1973, p. 709-723 (v. 2).

24. MALCHIODI, C.: PAPADIA, F. \& POUCHE, A. - Studio sperimentale sulla evoluzione della vascolarizzazione delle protesi arteriose sintetiche. Min. Chir. 26:1126, 1971. 
25. MARTELLI, A. \& REGGIANI, A. - Studio sperimentale sulle modificazioni dei vasa vasorum delle arterie renali dopo applicazione di um patch protesico. Arch. Ital. Urol. 37:446, 1965.

26. MARZOLI, G. P.; MEYER-BURGDCFF, G. \& JACQUET, G. H. - Sulle pseudo-cisti della parede arteriosa (con riferimento al quadro moriologico dei vasa vasorum). Chir. Ital. 14:290, 1962.

27. McCUNE, W. S.; THISTLETHWAITE, J. R.; KESHISHIAN, J. M. \& BLADES, B. - The nutrition of blood vessel grafts: an India ink injection study of their vascularization. Surg. Gynecol. Obst. 94:311, 1952.

28. McCUNE, W. S. \& BLADES, B. - 'The vascularization of arterial transplants. Bull. Soc. Int. Chir. 19:140, 1960.

29. NYLANDER, G. \& OLERUD, S. - The distribution of the vasa vasorum in the abdominal aorta and the vena cava inferior in dogs. Angiology 11:522, 1960.

30. OSGOOD, C. P.; DUJOVNY, M.; FAILLE, R.; BARRIONUEVO, P. J.; ZEA LONGA, E. \& MATTA, R. - Early scanning electron microcospic evaluation of microvascular maneuvers. Angiology 27:96, 1976.

31. PAGNANELLI, D. M.; PAIT, T. G.; RIZZOLI, H. V. \& KOBRINE, A. I. Scanning electron micrographic study of vascular lesions caused by microvascular needles and sutures. J. Neurosurg. 53:32, 1980.

32. RICHLING, B.; GRIESMAYR, G.; LAMETSCHWANDINER, A. \& SCHEIBLBRANDNER, W. - Endothelial lesions after clipping: a comparative study. J. Neurosurg. 51:654, 1979.

33. SCHLICHTER, J. G. - Experimental medionecrosis of the aorta. Arch. Path. 42:182, 1946a.

34. SCHLICHTER, J. G. - Studies on the vascularization of the aorta: the vascularization of the a.orta in the normal dcg. Amer. Heart J. 32:770, 1946b.

35. SCHLICHTER, J. G. \& HARRIS, R. - The vascularization of the aurta: a comparative study of the aortic vascularization of several species in health and disease. Am. J. Med. Sci. 218:610, 1949.

36. SCHONENBERGER, F. \& MULLER, A. - Ueber die Vaskularisation der Rinderaortenwand. Helv. Physiol. Pharm. Acta 18:136, 1960. Resumo em inglês.

37. SLAYBACK, J. B.; BOWEN, W. W. \& HINSHAW, D. B. - Intimal injury from arterial clamps. Am. J. Surg. 132:183, 1967.

38. STAUBESAND, J. - Ueber die Versorgung der arterienwand. Anat. Anz. 107:332, 1959. Resumo em inglês.

39. SUGITA, K. ; HIROTA, T.; IGUCHI, I. \& MIZUTANI, T. - Comparative study of the pressure of various aneurysm clips. J. Neurosurg. 44:723, 1976.

40. SUGIURA, A. - An experimental study on the vasa vasorum of the venous graft used in arterial replacement. Jap. Circ. J. 32:727, 1968.

41. THURSTON, J. B.; BUNCKE, H. J.; CHATER, N. L. \& WEINSTEIN, P. R. A scanning electron microscopy study of micro-arterial damage and repair. Plast. Reconstr. Surg. 57:197, 1976.

42. VIO, A.; REgGIANI, A.; GOZZetTI, G. \& PLATANIA, A. - Vantaggi dell'associazione china-goma liquida sintetica per dimostrare i vasa vasorum nel cane mediante iniezione intravitale. Boll. Scc. Ital. Biol. Sper. 40:859, 1964.

43. VIO, A.; GOZZETTI, G. \& REGGIANI, A. - Importanza dei vası vasorum nei processi di riparazione delle suture arteriose (studio sperimentale sul canne). Boll. Soc. Ital. Biol. Sper. 43:88, 1967.

44. WYATT, A. P.: ROTHNIE N. G. \& TAYLOR, G. W. - The vascularization of vein grafts. British J. Surg. 51:378, 1964.

Departamento de Cirurgia, Ortopedia e Traumatologia - Faculdade de Medicina de Ribeirão Prêto - Campus Universitário Monte Alegre - 14100 Ribeirão Prêto, SP - Brasil. 